\title{
Protección social, pandemia por COVID-19 y Agenda 2030 en Latinoamérica
}

\author{
Social Protection, pandemics and 2030 \\ Agenda in Latin America
}

\section{Nicole Elizondo 0.*}

Artículo de reflexión

Fecha de recepción: 31 de mayo de 2021

Fecha de aceptación: 21 de julio de 2021

\section{Para citar este artículo:}

Elizondo, N. (2021). Protección social, pandemia por COVID-19 y Agenda 2030 en Latinoamérica. Revista Análisis Jurídico-Político, 3(6), 127-146. https://doi.org/10.22490/26655489.4780

\section{Resumen}

La emergencia de la COVID-19 ha colocado en el centro de estudio la protección social y el cuidado de la red familiar, así como el anhelo de establecer una agenda universal e integral de cara al 2030. Los graves efectos causados por la pandemia hacen prever que el cumplimiento de los Objetivos de Desarrollo Sostenible (ODS) se verán limitados, producto del aumento en la pobreza y la desigualdad. Por tal razón, este artículo tiene como objeto indagar en la protección social en los países de Latinoamérica; en especial sobre los efectos de la pandemia en cuanto a transferencias, cobertura y prestaciones en

\footnotetext{
* Magíster en Gestión y Políticas Públicas, Universidad de Chile. Investigadora Centro UC de Familia, Pontificia Universidad Católica de Chile, Santiago de Chile. E-mail: nelizondo@uc.cl. Orcid: https://orcid. org/0000-0003-1984-0782.
} 


\section{ANÁLISIS

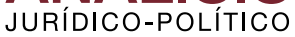

Chile. A continuación, se realizan reflexiones en torno al papel fundamental de la protección social para el cumplimiento de los ODS. Finalmente, se realizan algunas consideraciones en la materia.

Palabras claves: Agenda 2030, ODS, Protección Social, Latinoamérica, Chile

\section{Abstract}

The emergence of COVID-19 has placed social protection and care of the family network at the center of the study, as well as the desire to establish a universal and comprehensive agenda for 2030. The serious effects caused by the pandemic suggest that the fulfillment of the Sustainable Development Goals (SDG) will be limited as a result of the increase in poverty and inequality. For this reason, the objective of this article is to investigate social protection in Latin American countries, especially about the effects of the pandemic in terms of aid, coverage, and benefits in Chile. Then, reflections on the fundamental role of social protection for the fulfillment of the SDGs are presented. And finally, some considerations in this matter.

Keywords: Agenda 2030, SDG, Social Protection, Latin America, Chile

\section{INTRODUCCIÓN}

En Latinoamérica nos enfrentamos a vertiginosos procesos de cambios que se han acentuado con la llegada de la pandemia de la COVID-19. Así, los sistemas de protección social han experimentado desequilibrios económicos, sociales y ambientales que han desencadenado una respuesta rápida por parte de los estados. Hasta la actualidad, se han implementado políticas y medidas de emergencia como bonos y transferencias directas en apoyo al empleo y seguridad, a la educación a distancia, a los cuidados especiales hacia la población vulnerable, la asistencia y la ayuda económica a la familia, entre otras (Cepal, 2021).

Sin embargo, los prolongados periodos de confinamientos y restricciones han impactado en el sistema de protección social, en especial en la educación y cuidados de la infancia, la pérdida de 
ingresos familiares, así como los déficits en cobertura de prestaciones sociales y de la salud, que ya inquietaban a los gestores públicos previo a la llegada de la pandemia. Ello ha sido motivo de estudio con respecto al impacto que tendrá la pandemia en el cumplimiento de los Objetivos de Desarrollo Sostenible (en adelante ODS) adoptados por la Asamblea General de las Naciones Unidas desde el año 2015, tras el término de los Objetivos de Desarrollo del Milenio (2000-2015).

Los ODS contemplan 17 objetivos, 169 metas y 232 indicadores que tienen por objeto la superación de la pobreza y las desigualdades, la sostenibilidad y la prosperidad de cara al 2030 (ONU, 2015). En lo referido a la protección social, la denominada Agenda 2030 incluye la meta 1.3, que sostiene la aplicación a nivel nacional de sistemas y medidas apropiadas de protección social y que busca lograr ampliar la cobertura a la población pobre y vulnerable al 2030. La meta se complementa con los objetivos que guardan relación con la cobertura universal en salud (meta 3.8), la igualdad de género (meta 5.4), el trabajo decente y el crecimiento económico (meta 8.5), la mayor igualdad (meta 10.4) y la creación de instituciones eficaces, responsables y transparentes en todos los niveles (meta 16.6) (OIT, 2017).

A ello se agregan las recomendaciones de la Conferencia Internacional "Trabajando juntos por una Protección Social Universal en 2030", organizada por los miembros de la Alianza Global para la Protección Social Universal (USP2030), quienes reiteraron el compromiso por los llamados "pisos de protección social"1 (USP2030, 2019) de modo que, los sistemas sean considerados como un derecho humano esencial para la reducción de la pobreza y las desigualdades que afectan el bienestar social de las naciones.

Así, diversos estudios de organismos internacionales reiteran la contribución de la protección social al desarrollo económico, social y ambiental de los países (OCDE, 2009a; Banco Mundial, 2012; PNUD 2020a; UNICEF, 2012). Para el cumplimiento de la Agenda 2030, las

1 La Conferencia General de la Organización Internacional del Trabajo (OIT) define a los "pisos de protección social" como "conjuntos de garantías básicas de seguridad social definidos a nivel nacional que aseguran una protección destinada a prevenir o a aliviar la pobreza, la vulnerabilidad y la exclusión social" (Normativa Nº 202, inciso 2) (OIT, 2012). 
medidas de apoyo para robustecer los sistemas de protección social universal son clave para promover la justicia social, la seguridad social y el bienestar de la población (ONU, 2015). Por lo anterior, cabe preguntarse: ¿qué impacto tendrá la pandemia de la COVID-19 en el cumplimiento de los Objetivos de Desarrollo Sostenible en materia de protección social en Latinoamérica?

Las proyecciones pospandemia no son nada auspiciosas para la región Latinoamericana. La Cepal estima que la pobreza aumentaría en 209 millones, 22 millones de personas más que el año anterior. En tanto, la desigualdad medida a través del índice de Gini promedio podría incrementarse en $5.6 \%$ con respecto al 2019 (Cepal, 2021c). Por tales motivos, la emergencia de la COVID-19 vuelve a poner en el centro la protección social y cuidado de la red familiar, así como el anhelo de establecer una agenda universal e integral de cara al 2030.

Tomando en cuenta lo anterior, el presente artículo tiene como objeto indagar en la protección social en Latinoamérica y sus alcances en el cumplimiento de los ODS en el período postpandemia. De la protección social en Latinoamérica. Luego, se realiza una reflexión sobre los efectos de la pandemia COVID-19 en los sistemas de protección social tomando como caso de estudio a Chile. Finalmente, se realizan reflexiones en torno a las implicancias para el cumplimiento de la Agenda 2030 para posteriormente, exponer algunas consideraciones finales en la materia.

\section{Marco general: Protección social en Latinoamérica}

La literatura sobre protección social destaca los diversos aportes históricos y políticos, que dan cuenta de la evolución y desarrollo de los estados de bienestar o sistemas de protección social (Esping -Andersen y Myles, 2012; Lautier, 2006). En determinados periodos de tiempo, la vulnerabilidad e inseguridad social han incentivado a que los estados desarrollen redes de seguridad y protección a la población más vulnerable frente a procesos de inestabilidad económica, o bien para prevenir situaciones violentas contra la globalización (Rodrik, 1997), tales como precariedad social (desempleo, 
vejez, enfermedad, maternidad, empleo de las familias), incertidumbre económica y vulnerabilidad.

Se entiende por protección social "un derecho humano definido como un conjunto de políticas y programas diseñados para reducir y prevenir la pobreza y la vulnerabilidad en todo el ciclo de vida"2 (OIT, 2017, p. 2). Contempla beneficios amplios desde las prestaciones de maternidad, empleo, pensiones y salud a través de "una combinación de regímenes contributivos (seguro social) y de prestaciones no contributivas financiadas con impuestos (OIT, 2017, p. 2).

Comúnmente, la protección social es confundida con la política social o sectorial, sin embargo, es preciso señalar que estas políticas "se hacen cargo de la provisión de servicios sociales con los que se busca fortalecer el desarrollo humano" (Cepal, 2019, p. 16) mientras que, la protección social se encuentra "dirigida a asegurar un nivel básico de bienestar económico y social a todos los miembros de la sociedad" (Cepal, 2019, p. 16).

El enfoque de protección social se traduce en transferencias condicionadas, subsidios y/o asistencia social destinada a la población con mayor vulnerabilidad (Midgley, 2012). De modo que los sistemas de protección social son considerados elementales para la reducción de la pobreza y desigualdades (Canudas, 2002; Cepal, 2016; Sojo, 2017). Destacan la implementación de programas de transferencias monetarias y transferencias condicionadas sobre alimentación, asistencia escolar o controles médicos de salud, las cuales están dirigidas en especial a la familia y a la infancia en situación de pobreza y pobreza extrema (Cepal, 2019).

A nivel mundial, las estimaciones estadísticas de la Organización Internacional de Trabajo (OIT) sostienen que un $71 \%$ posee cobertura parcial o no posee protección social mientras que, solo un $29 \%$ declara que se encuentra protegido por un sistema de seguridad social integral (OIT, 2017, p. 32). De hecho, un $45 \%$ se encuentra cubierta con al menos un beneficio de protección social (OIT, 2017) registrándose en América Latina y el Caribe la cifra de $61.4 \%$ (OIT, 2017).

2 Cabe agregar que la protección social, o la seguridad social, está consagrada como tal en la Declaración Universal de Derechos Humanos, 1948, el Pacto Internacional de Derechos Económicos, Sociales y Culturales, 1966, y en otros instrumentos fundamentales de las Naciones Unidas sobre los derechos humanos (OIT, 2017: 216). 
En América Latina, los sistemas de protección social han seguido los modelos europeos basados en objetivos universalistas de derechos sociales (Gomariz, 2007; Vuolo, 2009); sin embargo, el tránsito hacia sistemas que aseguren un ingreso básico fue truncado debido al llamado fracaso de las "políticas focalizadas" (Lautier, 2006). Lautier, sostiene que dichas políticas "no sólo son costosas - por cabeza-, sino que eliminar muy esporádicamente las causas de lo que supuestamente deben combatir" (Lautier, 2006, p. 657).

Sin embargo, dichas políticas han permitido el desarrollo de planes y programas para la superación de la pobreza (Merrien, 2013). Durante la década del 90, organismos internacionales tales como Cepal, OIT y el BID han aportado con estudios a numerosos países con el fin de promover la implementación de políticas sociales y reformas estructurales para asegurar los llamados nuevos pactos sociales que sitúan al centro la equidad y la cohesión social (Lautier, 2006; Cepal, 2006).

No obstante, es preciso señalar que la región posee limitaciones en cuanto a la implementación de sistemas de protección social inclusivos, universales y equitativos (Duhau, 1997; Draibe y Riesco, 2009). A diferencia de las democracias occidentales que cuentan con sólidos estados de bienestar; las políticas públicas y la red de protección social han sufrido alteraciones producto de la falta de continuidad y coherencia institucional. Históricamente, "han evolucionado de manera fragmentada y estratificada, dando origen a la creación de déficits de cobertura y desigualdades en el alcance y la suficiencia de sus prestaciones" (OIT, 2017, p. 146).

Para ello, resulta clave el financiamiento y la política fiscal, pues, en promedio, los países con ingreso per cápita más alto poseen mejores resultados en el cumplimiento de los ODS en comparación con los países de ingresos bajos, quienes por el tipo de economía emergente desembolsan menores recursos en áreas como la educación, salud e infraestructura - ejes claves para el logro de los ODS- (Gaspar et ál., 2019).

Así, en América Latina el gasto en protección social fue de $4.1 \%$ del PIB para el año 2018 (Cepal, 2021b), siendo los países con mayor gasto: Brasil (12.7\%), Argentina (11.1\%), Uruguay $(7.2 \%)$, Chile $(6.1 \%)$ y Colombia $(6.1 \%)$, en contraposición, los países que registran menores niveles de gasto fueron: Nicaragua $(0.6 \%)$, 
Honduras $(0.7 \%)$, Ecuador (1.2\%), Guatemala (1.3\%) y Panamá $(1.3 \%)$ (Cepal, 2021d).

De este modo, Antía (2018) sostiene que en el continente es posible distinguir dos conjuntos de países en lo referido a las políticas de bienestar social en función de estado, mercado y familia. El primero se caracteriza por mayores niveles en las coberturas social, aun cuando "sus regímenes de políticas sociales presentan un alto nivel de segmentación y de presencial del mercado" (Antía, 2018, p. 200) - se encuentra: Argentina, Brasil, Chile, Costa Rica, Uruguay y México-. En el caso del segundo conjunto, destaca la ausencia de ofertas programáticas y la insuficiente presencia del mercado de trabajo en la familia - lo componen: Bolivia, Colombia, Ecuador, El Salvador, Guatemala, Honduras, Nicaragua, Panamá, Paraguay y Perú-.

Así, los países de la región determinan el gasto social con en base el resultado del crecimiento económico y la estabilización del mercado. En retrospectiva, los sistemas de protección social en la región destacan por la implementación de políticas dirigidas a combatir la pobreza, las que han contribuido a su disminución de manera sostenida. Si en 1990 la pobreza era de $51.2 \%$ y la pobreza extrema de $15.5 \%$, hacia el 2019, esta disminuyó a $30.5 \%$ y $11.3 \%$ respectivamente. Tendencia que con la llegada de la pandemia ha aumentado en $33.7 \%$ y $12.5 \%$ respectivamente (Cepal, 2021).

Junto con lo anterior, se comenzó a diversificar las políticas sociales en materias de educación, salud, vivienda y cuidados familiares, con ello la ampliación de cobertura, acceso y beneficiarios (Brodersohn, 1992; Cohen y Franco, 2006). De este modo, según los datos del Informe Mundial sobre la Protección Social 2017-2019 (OIT, 2017) destaca la protección social universal de los niños (Argentina, Brasil, Chile), cobertura universal a madres de recién nacidos (Canadá y Uruguay), cobertura universal de personas con discapacidad (Brasil, Chile, Estados Unidos, Uruguay), la cobertura universal de pensiones de vejez (Argentina, Bolivia, Canadá, Estados Unidos y Trinidad y Tobago) (OIT, 2017, p. 131).

De este modo, se han constituido marcos legislativos nacionales que han permitido establecer, poco a poco, sistemas de prestaciones sociales; así, se puede resaltar el Programa Bolsa de Familia (Brasil), el Programa Oportunidades (México), Chile Solidario (Chile) y el Programa Más Familias en Acción (Colombia). Icónicos programas 
sociales que han contribuido al mejoramiento de la educación, salud y nutrición (Cepal, 2019). En lo específico, es posible destacar el aumento de la matrícula y asistencia escolar, disminución de la tasa de repetición y abandono escolar, mayor regularidad de controles de salud y nutricional a la infancia, entre otros.

Sin embargo, "la extensión de la cobertura legal no garantiza por sí misma la cobertura efectiva de la población o la mejora de la calidad y el nivel de las prestaciones" (OIT, 2017, p. 5). Si bien los avances hacia la universalidad se encuentran en la dirección adecuada, los sistemas de protección en la región presentan déficits importantes en cuanto a la cobertura, así como con respecto al financiamiento y la extensión en el tiempo de los programas sociales.

A ello se agrega que, la limitada oferta programática en protección social ha impedido que grupos de clases medias se desarrollen social y económicamente a largo plazo. Si bien, la protección social a este grupo ha sido un tema recurrente de discusión (Schlogl y Sumner, 2014) conviene señalar que la problemática no ha sido adecuadamente abordada por la institucionalidad, lo que ha generado foco de frustración y molestia por generaciones especialmente jóvenes, trabajadores informales, independientes, mujeres jefas de hogar.

En definitiva, los sistemas de protección en Latinoamérica han progresado y contribuido al progreso y bienestar social; sin embargo, las disparidades históricas que mantiene la región en términos de desigualdad y pobreza mantienen rezagos en cuanto a seguridad social universal, en especial para la población de mujeres, jóvenes e indígenas, quienes se han visto más afectados por la pandemia de la COVID-19 (Cepal, 2021).

\section{IMPLICANCIAS PARA LA PROTECCIÓN SOCIAL FRENTE LA PANDEMIA DE LA COVID-19}

La irrupción de la pandemia de la COVID-19 en Latinoamérica ha acrecentado las disparidades de la protección social, en especial sobre lo relativo a la pobreza y las desigualdades históricas en la región (ONU, 2020). Así, los países han dispuesto de forma urgente la adecuación de la institucionalidad pública para asegurar el bienestar y el desarrollo social (Brewer y Gardiner, 2020). 
En términos generales, los países han desplegado una serie de medidas de emergencia destinadas mayoritariamente a la población vulnerable (Lustig et ál., 2020), siendo la implementación de transferencias monetarias la fórmula más recurrente (Cecchini et ál., 2015). Según datos del último informe "Panorama Social" de la Cepal, durante el año 2020 se adoptaron 263 medidas no contributivas de protección social en 32 países de la región. En total, las medidas relacionadas con el COVID-19 representan el 49,4 \% en cobertura materializadas en transferencias monetarias y en especie; en tanto "para el acceso garantizado a servicios básicos, 127 fueron adoptadas en América del Sur, 74 en los países del Caribe y 62 en Centroamérica" (Cepal, 2021, p. 133).

Así destacan iniciativas como "Ingreso Familiar de Emergencia" (Argentina), el "Auxilio Emergencial" (Brasil), el "Bono de Emergencia COVID-19" y el "Ingreso Familiar de Emergencia" (Chile), el "Ingreso Solidario" (Colombia), el "Bono de Compensación" (El Salvador) o bien, la entrega de canastas básicas de alimentación y voucher para la adquisición de estos - tales como "Alimentos para Chile" (Chile), "Alimentos en Casa" (Costa Rica), "Programa de Seguridad Alimentaria Nangareko" (Paraguay)—.

De manera similar, se han establecido normativas de flexibilidad del pago servicios básicos — agua y energía eléctrica- en los que se otorgan subsidios como sucedió en los países de Chile, Costa Rica y El Salvador; en tanto en algunos casos se aplicó el congelamiento de las tarifas de telefonía fija, móvil e internet (Argentina), la exención de impuestos de servicios de voz e internet móvil (Colombia) o bien, la implementación de bonos para la electricidad o gas (Perú).

En el 2020 se destinaron alrededor de 86214 millones de dólares para el financiamiento de medidas no contributivas que se destinaron a planes de emergencia para las familias de menores ingresos a través de transferencia monetaria y/o en especie (Cepal, 2021). Dichas medidas de emergencia han sido inéditas en la región y han amainado las profundas consecuencias socioeconómicas para las familias, ya sea través de transferencias monetarias, en especie (alimentos, medicamentos, mascarillas y productos de aseo), suministros de servicios básicos, protección al empleo formal y apoyos directos tales como medidas tributarias, facilidades de pagos de créditos e hipotecas, entre otros (Cepal, 2021). 
El rápido despliegue de programas y políticas de emergencia se debe a que los gobiernos "contaban ya con sistemas de información y con canales para entregar los pagos, con que los gobiernos pudieran identificar, incorporar y atender a un sector de la población" (PNUD, 2020b, p. 6). Sin embargo, dado las disparidades propias de los países - fragmentación y desigualdades en los sistemas de protección social (Cepal, 2021)—, los beneficios y apoyos excluían a las capas medias y aquellos en condición de informalidad, los cuales no se encontraba identificados previo a la pandemia.

Con respecto al gasto público social, previo a la llegada de la pandemia de la COVID-19, el gasto social en América Latina y el Caribe se caracterizó por la diversidad en su composición: “ocho países asignan menos del $10 \%$ del PIB al gasto público social del gobierno central y tres superaron el $17 \%$ en 2019, con montos que a su vez varían entre menos de 200 y más de 2500 dólares per cápita al año" (Cepal, 2021, p. 33). De hecho, a nivel general en el año 2019, los programas de transferencias condicionadas solo tenían una cobertura de 18,5\% promedio en la región (Cepal, 2021, p. 31).

Según explicitan los autores Blofield, Giambruno y Filgueira (2020) los gastos en políticas sociales es de $0,3 \%$ del PIB regional, lejos de los 2,7 \% de los países de la OCDE. Ello, refuerza los datos de cobertura en disminución desde 2013 (Lavinas, 2021) lo que, se condice con los períodos de ralentización económica que experimentó la región (Cepal, 2020).

Sin embargo, el desempeño de los países en el manejo de la pandemia ha sido deficiente (Lavinas, 2021) en términos de la diversidad de las medidas de apoyo, los montos, la frecuencia de entrega y la cobertura (PNUD, 2020b). Ello desencadenó en malestar de la sociedad, como quedó evidenciado en las sucesivas protestas en Colombia o Brasil, donde las clases medias, desempleados o trabajadores informales han quedado rezagados de las ayudas sociales destinadas a la emergencia y donde, además, la población acusa que las medidas de apoyo destinan bajos montos de transferencias reales (Lavinas, 2020).

Así, parece interesante como caso de estudio Chile, país que ha desembolsado alrededor de 8,2\% del PIB en gastos adicionales e ingresos percibidos, en tanto ha destinado $1,8 \%$ en préstamos y garantías (FMI, 2021) convirtiéndose en el segundo país de Latinoamérica con 
mayor gasto fiscal durante la pandemia después de Brasil. Sin embargo, pese a las destacadas medidas en beneficio a la protección social de las familias más vulnerables; el apoyo no ha llegado de manera extensiva a la población de clase media, lo cual desencadenó sucesivos ajustes institucionales al sistema de protección producto de la pandemia.

\subsection{El caso de Chile}

En Chile, a partir de la promulgación del estado de emergencia por la pandemia del coronavirus (Decreto n. ${ }^{\circ} 104$, de 18 de marzo de 2020) y la aplicación de la alerta sanitaria por parte del Ministerio de Salud (Decreto n. ${ }^{\circ} 4$, de 08 de febrero de 2020) se establecieron una serie de medidas de restricción de movimiento y limitaciones a las actividades diarias de las personas. La institucionalidad pública, a través del Ministerio de Hacienda y el Ministerio de Desarrollo Social y Familia, lideraron los planes financieros y sociales para los hogares afectados por la pandemia.

En primer lugar, el Ministerio de Hacienda estableció el llamado "Plan Económico de Emergencia" (2020) con el objeto de implementar medidas en apoyo a las empresas pymes, trabajadores y familias. Sin embargo, tras una negociación entre el Gobierno y partidos políticos de oposición se estableció el "Marco de Entendimiento para un Plan de Emergencia por la Protección y Reactivación", que contiene recursos por 12000 millones de dólares durante 24 meses.

En segundo lugar, el Ministerio de Desarrollo Social y Familia desplegó una serie de medidas de emergencia en apoyo a las familias de mayor vulnerabilidad. Se encuentran la creación de nuevos beneficios tales como, el Bono de Emergencia COVID-19, el Fondo Solidario a Municipios, el Ingreso Familiar de Emergencia (IFE), y su aplicación más reciente el IFE Ampliado a los hogares del $100 \%$ del Registro Social de Hogares (RSH), el Bono Clase Media y el préstamo Solidario Clase Media.

En transferencias directas, destaca la implementación del llamado Ingreso Familiar de Emergencia (IFE), desde junio de 2020 el beneficio contemplaba al $60 \%$ más vulnerable del RSH y los montos se encontraban sujetos a la cantidad de personas que integran el hogar, así como la situación socioeconómica de la familia. A raíz de las 
críticas por los requisitos y el acceso para las familias se implementó el IFE 2.0, que eliminó el requisito de vulnerabilidad, flexibilizó la acreditación de los ingresos formales del hogar y aseguró el pago por al menos seis meses.

Posteriormente, para los meses de enero, febrero y marzo del 2021, se crea el IFE COVID Cuarentena e IFE COVID Transición, beneficios destinados al $60 \%$ más vulnerable del RSH, por un monto de hasta \$100 000 PCL por persona y condicionado a la situación sanitaria de la comuna (cuarentena o transición) de acuerdo con el Plan Paso a Paso dispuesto por el Ministerio de Salud. En tanto, en abril de 2021 se anunció el IFE Ampliado para los hogares que se encuentran en el $80 \%$ de vulnerabilidad y contempla el pago de $\$ 100000$ PCL por persona por un periodo de tres meses (mayo, junio y julio). A diferencia de su versión anterior, el beneficio se entrega independientemente de la situación sanitaria de la comuna y sin tope máximo de ingreso familiar. A modo de ejemplificar, el IFE Ampliado correspondiente a abril del 2021 tuvo una cobertura de 5336001 hogares correspondientes a 12121438 millones de beneficiarios, lo que implica el aumento promedio del doble de beneficiarios en comparación a los IFE anteriores.

Sumadas a las transferencias directas, en el país se implementaron medidas de contingencias para contener los efectos económicos de la crisis sanitaria de la COVID-19, tales como la ampliación del subsidio de arriendo, subsidios para las cuentas básicas (luz, agua e internet), subsidios para familias vulnerables ${ }^{3}$, la postergación del pago de los créditos hipotecarios y universitarios, y el aplazamiento del pago de contribuciones, la campaña de entrega de cajas de alimentación ${ }^{4}$, entre otros ${ }^{5}$.

A nivel comparado, Chile se ubica como el segundo país que más gasto social ha realizado después de Brasil durante la pandemia

3 Dentro de los destacados, Préstamo Estatal Solidario que tiene por objeto $70 \%$ de la caída de ingresos formales mensuales del solicitante, así como el Subsidio al Empleo (Fondo COVID) que establece con el objetivo de incentivar el regreso de los trabajadores suspendidos durante la pandemia, así como promover la contratación de nuevos trabajadores para las empresas.

4 La campaña "Alimentos para Chile", que entrega canastas con alimentos en su primera etapa a cerca de 3 millones de familias de sectores bajos y medios - distribuidas a nivel nacional-.

5 Cabe agregar, disposiciones que complementan las medidas tales como prórroga del vencimiento de licencia de conducir, carné de identidad, pasaporte, entre otros. 
(FMI, 2021). Según datos de la segunda ronda de la Encuesta Social COVID-19 (2021), los hogares que declaran recibir transferencias directas o bienes y servicios de parte del estado alcanzaron un 52,4 \% en la medición.

Sin embargo, a nivel territorial existen disparidades con respecto a la percepción de los beneficios en la población en general, pues las transferencias y medidas destinadas a las familias se encuentran condicionadas a determinadas características (número de integrantes, tipo de vulnerabilidad, inscripción en el Registro Social de Hogares, entre otros). La misma encuesta sostiene que en 2019, un $16,5 \%$ de los hogares percibía que los ingresos "no les alcanzaban" para financiar sus gastos, en tanto en noviembre-diciembre de 2020 la cifra aumentó al 31 \% (Ministerio de Desarrollo Social y Familia y PNUD, 2021).

Si bien existe una valoración positiva de las transferencias y medidas de emergencia focalizadas en la población vulnerable, cabe destacar la opinión generalizada sobre las exigencias y limitaciones para el acceso a los beneficios. Dichos argumentos fueron el refuerzo para la aprobación histórica de los tres proyectos de ley para el retiro del $10 \%$ del fondo de pensiones - Ley n. ${ }^{\circ} 21.248$ (publicada el 30 de julio de 2021), Ley n. 21.295 (publicada el 10 de diciembre de 2021), Ley n. 21.330 (publicada el 28 de abril de 2021)—.

Tras el tercer retiro del $10 \%$ en abril del año 2021, la crisis institucional derivó en la llamada "Agenda de los Mínimos Comunes" entre el gobierno de Sebastián Piñera y el Congreso, el que tuvo como objeto trabajar una agenda colaborativa que permita implementar de manera urgente mejores ayudas destinadas a las familias y pymes afectadas por la pandemia. Así, en mayo de 2021, el gobierno ingresó dos proyectos de ley con discusión inmediata: el que "Establece nuevas medidas tributarias para apoyar a las micro, pequeñas y medianas empresas, por la crisis generada por la enfermedad COVID-19" (Boletín 14278-05) así como el proyecto de ley que "Modifica la ley n. ${ }^{\circ} 21.289$, de Presupuestos del Sector Público correspondiente al año 2021, y la ley n. ${ }^{\circ} 21.230$, que concede un Ingreso Familiar de Emergencia" (Boletín 14277-31).

Respecto está última, se establece la ampliación del Ingreso Familiar de Emergencia al $100 \%$ del Registro Social de Hogares - excluyendo a las personas del $10 \%$ de más altos ingresos- para los meses de 
junio, julio, agosto y septiembre. El nuevo IFE beneficiará a aproximadamente 14 millones de personas equivalente a cerca de 7 millones de hogares (Dirección de Presupuestos, 2021) con un costo fiscal de 2890 millones de dólares.

\section{Implicancias para el CUMplimiento de la Agenda 2030 POSPANDEMIA COVID-19}

Como ha quedado demostrado, los paquetes de protección social y estímulo fiscal dirigidos a las familias más vulnerables resultan esenciales para aliviar las graves consecuencias de la crisis del coronavirus. En la región, los sistemas de protección social catalogados como débil y fragmentados (Cepal, 2021) han mantenido problemas en su cobertura y prestaciones, lo que ha implicado importantes brechas dentro de los propios países de la región; "por un parte, ha aumentado la demanda urgente de protección social y, al mismo tiempo, los recursos gubernamentales han resultado mermados por la disminución de los ingresos fiscales y de las contribuciones al seguro social" (OIT, 2020, p. 2).

Por ello, el impacto del COVID-19 en el cumplimiento de los ODS es preocupante, toda vez que según estimaciones la pobreza ha marcado su máximo en 20 años a causa de la pandemia (Cepal, 2021), lo que ha tenido relación directa con el deterioro de las condiciones de bienestar: desincentivo a la participación laboral —especialmente el femenino y de jóvenes-, brechas del aprendizaje en la educación, limitaciones en la cobertura del sistema de salud, entre otros.

La crisis socioeconómica ocasionada por la pandemia dejará huellas por más de una década en la región (Jordà et ál., 2020), incluso se está comenzando a discutir de la posible década perdida en Latinoamérica (Powell y Valencia, 2020). En la pospandemia, los sistemas de protección social estarán afectos a importantes costos en la igualdad; sin embargo, es una oportunidad en cuanto a la "creación de un verdadero Estado de bienestar, tarea largamente postergada en la región" (Cepal, 2021, p. 1), así como fortalecer la distintas áreas de la Agenda 2030.

Como queda explícitamente explorado a lo largo del artículo, en la actualidad los Estados han desplegado una gran cantidad de medidas 
de emergencias temporales a las familias más vulnerables. Sin embargo, es innegable que en los próximos años se aplicarán medidas de austeridad y control del gasto público que afectarán la cobertura de la protección social. Según estimaciones, "los países en desarrollo necesitarán realizar una inversión adicional de 1,2 billones de dólares de los Estados Unidos, lo que equivaldría al 3,8 por ciento de su producto interno bruto (PIB), para subsanar la brecha anual en el financiamiento en 2020" (OIT, 2020, p. 1).

De este modo, para evitar la ralentización del cumplimiento de la Agenda 2030, así como la recomendación número 202 sobre los pisos de protección social de la OIT, resultan cruciales las recomendaciones realizadas por Durán-Valverde et ál. (2020): por un lado, extender la cobertura de la seguridad social y los ingresos por cotizaciones sociales; por otro lado, incrementar la AOD y las transferencias.

De cara al 2030, los sistemas protección social en la región tendrán que lidiar con los graves efectos de la pandemia, empero, grandes desafíos le esperan. Es decir, "son necesarios mayores esfuerzos para necesarios mayores esfuerzos para asegurar que los sistemas de protección social estén bien institucionalizados y reconocidos en la ley y en las estrategias nacionales de desarrollo, que cuenten con una base de financiación estable y sostenible, y que su administración y gestión sean eficaces y eficiente" (OIT, 2019, p. 192).

En definitiva, invertir en protección social no solo mejorará en el cumplimiento de la Agenda 2030 sino que marcará la recuperación tras la pandemia, lo cual consolida los sistemas por las próximas décadas para Latinoamérica.

\section{Reflexiones finales}

Desde su implementación en el 2015, la Agenda 2030 se ha caracterizado por su integralidad y variedad de objetivos de intervención frente a los problemas globales. A diferencia de los Objetivos de Desarrollo del Milenio adoptado en el 2000, los ODS abarcan múltiples objetivos (17), metas (169) e indicadores (232) bajo la promesa de avanzar hacia la sostenibilidad social, económica y medioambiental al 2030. 
Previo a la llegada de la pandemia de la COVID-19, los países de Latinoamérica se encontraban trabajando en el cumplimiento de las metas e indicadores para la consecución de la Agenda 2030; esto, pese al contexto de ralentización económica que ha acompañado la región de manera continuada en la última década. Posteriormente, a raíz de los efectos de la pandemia, los sistemas de protección social han experimentado desequilibrios económicos, sociales y ambientales que han desencadenado en una rápida respuesta por parte de los Estados.

Sin embargo, el cumplimiento de los ODS y la Agenda 2030 han sido cuestionados debido a las luces y sombras que ha traído consigo la pandemia. Por un lado, ha comenzado el debate sobre la reconfiguración de sociedades más sostenibles; por otro, ha aumentado las brechas en cuanto a la vulnerabilidad de la población respecto a las condiciones de bienestar y cohesión social, opacando los logros de los últimos años en materia educacional, igualdad de género y salud.

Toca ahora responder la pregunta central del artículo: ¿qué impacto tendrá la pandemia de la COVID-19 en el cumplimiento de los Objetivos de Desarrollo Sostenible en materia de protección social en Latinoamérica? En mi opinión, frente a la catástrofe social y económica, la visión holística de los ODS ofrece acciones sostenibles para la gobernanza y las políticas públicas pospandemia. Como se pudo observar a lo largo del artículo, los sistemas de protección social en América Latina se han caracterizado por su fragmentación, alta focalización y vulnerabilidad a los cambios sociopolíticos de los estados; elementos que en algunos casos se han deteriorado producto de la pandemia.

En ese contexto, la Agenda 2030 sin duda se verá afectada en mayor medida en los países en desarrollo, En particular en lo referido al trabajo decente, la igualdad de género, la nutrición, la salud mental y el medio ambiente. Por tanto, la importancia de la transversalidad de la política social resulta clave para la coordinación, diseño e implementación de programas hacia los grupos vulnerables y capas medias de la población.

Por tanto, para el cumplimiento de los ODS y la superación de los efectos pospandemia, los estados latinoamericanos tienen la oportunidad de introducir cambios en las políticas sociales focalizadas 
de modo de ampliar los beneficiarios y, a largo plazo realizar reestructuraciones a los sistemas de protección social como imperativo para lograr el desarrollo, inclusión y cohesión social.

Las políticas públicas, presupuestos, instituciones y marcos legales requieren de mejoras en la coherencia e integración de los ODS. La inversión en la recuperación resulta fundamental para la movilización de recursos de manera ágil y oportuna en vista de los compromisos y secuelas de la pandemia en cuanto al desempleo, salud, educación y pobreza. Pese a las proyecciones de austeridad y control del gasto público que eventualmente afectarían el financiamiento de programas de protección social y a las diferencias significativas de los países de la región; las transferencias y programas sociales son clave para fortalecer el cumplimiento de los ODS.

La complejidad del contexto actual conlleva desarrollar nuevas investigaciones empíricas que den cuenta del diagnóstico, impactos e implicancias de la COVID-19 sobre los ODS y los sistemas de protección social. A medida que nos acercamos al 2030, contar con este tipo de investigaciones en perspectiva temporal puede ofrecer nuevas aristas del fenómeno en cuestión especialmente en la gobernanza e instituciones (ODS 16) y las desigualdades (ODS 10).

Finalmente, resulta fundamental que los estados realicen ajustes y adaptaciones de políticas de modo de asegurar y garantizar el acceso a beneficios y asistencia social. Los sistemas de protección social se encuentran con importantes desafíos tras la pandemia de la COVID-19, por lo que es un imperativo la inversión en políticas centradas contra la pobreza, la desigualdad y la exclusión social con el fin de preservar los derechos humanos de la población.

\section{Referencias}

Antía, F. (2018). Regímenes de política social en América Latina: una revisión crítica de la literatura. Desafíos, 30(2), 193-235.

Banco Mundial. (2012). Resilience, equity and opportunity: The World Bank's Social Protection and Labor Strategy 2012-2022. Washington D.C.

Brewer, M. y Gardiner, L. (2020). The initial impact of COVID-19 and policy responses on household incomes. Oxford Review of Economic Policy, graa024. https: / / doi.org/10.1093/oxrep/graa024 


\section{ANÁLISIS \\ JURÍDICO-POLÍTICO}

Blofield, M., C. Giambruno y F. Filgueira. (2020). Policy expansion in compressed time: assessing the speed, breadth and sufficiency of post-COVID-19 social protection measures in 10 Latin American countries. Serie Políticas Sociales, $N^{o} 235$ (LC/TS.2020/112), Santiago, Comisión Económica para América Latina y el Caribe (Cepal).

Brodersohn, V. (1992). De las estrategias de desarrollo social a los programas de compensación social. Buenos Aires: OEA, Centro Interamericano para el Desarrollo Social.

Canudas, R. (2002). Inclusión social. Perspectiva de las estrategias para la reducción de la pobreza. En Inclusión social: Una perspectiva para la reducción de la pobre$z a$. INDES-BID. https: / / bit.ly/ ЗepejeC

Cecchini, S., Filgueira, F., Martínez, R. y Rossel, C. (2015). Towards Universal Social Protection. Latin American Pathways and Policy Tools. ECLAC: Santiago. ssrn. com $/$ abstract $=2699403$

Cepal. (2021). Panorama Social de América Latina 2020. https: / / bit.ly /3kpp4l2

Cepal. (2021b). América Latina (17 países): gasto en protección social del gobierno central, 2000-2018 (En porcentajes del PIB). Base de datos de inversión social en América Latina y el Caribe. https: / / bit.ly/3icX6G1

Cepal. (2021c). Secretaria Ejecutiva de Cepal recalca trabajo conjunto con la OCDE para apoyar el desarrollo sostenible de los países de América Latina y el Caribe. https:/ / bit.ly/3enXZuK

Cepal. (2021d). América Latina (17 países): gasto en protección social del gobierno central, $2018^{a}$ (En porcentajes del PIB). Base de datos de inversión social en América Latina y el Caribe. https:/ / bit.ly/2UJxTLz

Cepal. (2020). Informe sobre el impacto económico en América Latina y el Caribe de la enfermedad por coronavirus (COVID-19). https: / / bit.ly/3xJ4zn5

Cepal. (2019). Protección social universal en América Latina y el Caribe. Textos seleccionados 2006-2019. https: / / bit.ly/3wGep7P

Cepal. (2016). Desarrollo social inclusivo: una nueva generación de politicas para superar la pobreza y reducir la desigualdad en América Latina y el Caribe. https://bit. ly / 2UPYUgm

Cepal. (2006). La protección social de cara al futuro: Acceso, financiamiento y solidaridad, Santiago de Chile. https: / / bit.ly/3rbKq6N

Cohen, E. y Franco, R. (2006). Los programas de transferencias con corresponsabilidad en América Latina: Similitudes y diferencias", en Cohen, E. y Franco, R. (coords.). Transferencias con corresponsabilidad: Una mirada latinoamericana. México: Secretaría de Desarrollo Social, pp. 23-84.

Draibe, S. y Riesco, M. (2009). El Estado de bienestar social en América Latina. Una nueva estrategia de desarrollo. Documento de Trabajo No. 31, Madrid: Fundación Carolina.

Duhau, E. (1997). Las políticas sociales en América Latina: ¿Del universalismo fragmentado a la dualización?". Revista Mexicana de Sociología, 59(2),185-207.

Durán, F., Jiménez, J., Muzaffar, T. y Elizondo, H. (2020). Financing Gaps in Social Protection: Global Estimates and Strategies for Developing Countries in Light of COVID-19 and Beyond. Documento de trabajo. Ginebra: Oficina Internacional del Trabajo. 
Esping-Andersen, G. y Myles, J. (2012). The Welfare State and Redistribution. https:/ / bit.ly / 3xJSVZ8

FMI (2021). Fiscal Monitor: Database of Country Fiscal Measures in Response to the COVID-19 Pandemic. https: / / bit.ly /3xJ568z

Gaspar, V., Amaglobeli, D., Garcia-Escribano, M., Prady, D., Soto, M., e International Monetary Fund. (2019). Fiscal policy and development: Human, social, and physical investment for the SDGs. https: / / bit.ly/3rbCOks

Gomariz, E. (2007). Sistema político y políticas públicas en América Latina. Revista Reforma y Democracia, 38, 91-112.

Jordà, O., Sanjay R. Singh y Alan M. Taylor. (2020). La larga resaca económica de la Pandemia. Revista Finanzas y Desarrollo, 57(2), 12-15. https: / / bit.ly/3rd9xWE

Lautier B. (2006). Condiciones políticas para la universalización de la protección social. En Le Bonniec, Y. y Rodríguez, O. (eds.). Crecimiento, equidad y ciudadanía. Hacia un nuevo sistema de protección social (pp. 257-691). CID-Universidad Nacional de Colombia.

Lavinas, L. (2021). Latin America at the crossroads yet again: what income policies in the post-pandemic era?. Canadian Journal of Development Studies, 42, 1-2.

Lavinas, L. (2020). The Collateralization of Social Policy by Financial Markets in the Global South. En P. Mader, D. Mertens, and N. Van der Zwan (edse). The Routledge International Handbook of Financialization (pp. 312-323). Routledge.

Lustig, N., V. Martínez, F. Sanz, y S. Younger. (2020). The impact of COVID-19 lockdowns and expanded social assistance on inequality, poverty and mobility in Argentina, Brazil, Colombia and Mexico. Tulane University, CEQ Institute.

Merrien, F. (2013). La protection sociale comme politique de développement: un nouveau programme d'action international. International Development Policy. Revue internationale de politique de développement. Revue internationale de politique de développement, 4(2), 68-88.

Midgley, J. (2012). Social protection and social policy: key issues and debates. Journal of Policy Practice, 11,8-24.

Ministerio de Hacienda de Chile. (2021). Plan de Emergencia y Plan de Recuperación: Avances y Cumplimiento. https: / / bit.ly/3ko9BSf

Ministerio de Desarrollo Social y Familia y PNUD. (2021). Encuesta Social COVID-19. Segunda ronda noviembre 2020. https: / bit.ly/2VIUY1n

OCDE. (2009a). Promoting pro-poor growth: Social protection. Comité de Asistencia para el Desarrollo.

OIT. (2020). Foco en la protección social. Brechas en el financiamiento de la protección social: estimaciones mundiales y estrategias para los países en desarrollo en el contexto de la crisis de la COVID-19 y más allá. https: / / bit.ly/3z24VW3

OIT. (2019). Declaración del Centenario de la OIT para el futuro del trabajo. https: / / bit. ly $/ 3 \mathrm{kusvHb}$

OIT. (2017). Informe Mundial sobre la Protección Social 2017-2019: La protección social universal para alcanzar los Objetivos de Desarrollo Sostenible. Oficina Internacional del Trabajo - Ginebra: OIT.

OIT. (2012). R202 - Recomendación sobre los pisos de protección social, 2012 (202). https: / / bit.ly/3xNxab0 


\section{ANÁLISIS \\ JURÍDICO-POLÍTICO}

ONU. (2020). El impacto del COVID-19 en América Latina y el Caribe. https://bit. ly/3kpqDz6

ONU. (2015). Resolución aprobada por la Asamblea General el 25 de septiembre de 2015 70/1. Transformar nuestro mundo: la Agenda 2030 para el Desarrollo Sostenible. https:/ / bit.ly/3hIzV7D

Powell, A. y Valencia, O. (2020, 15 de octubre). ¿Cómo evitar una nueva década perdida para América Latina y el Caribe? Ideas que Cuentan. BID. https:/ / bit. ly/3B5JeX0

PNUD. (2020a). Lecciones del COVID-19 para una agenda de sostenibilidad en América Latina y el Caribe. Serie de Documentos de Política Pública. PNUD.

PNUD. (2020b). Respuestas para enfrentar la pandemia en América Latina y el Caribe: el uso de programas de transferencias monetarias y de sistemas de información de protección social. PNUD.

USP2030. (2019). Unidos para lograr la protección social universal en 2030 (USP 2030) -Llamados a la acción-. https: / / bit.ly/3wDEaFS

Rodrik, D. (1997). Globalization, Social Conflict and Economic Growth Prebisch Lecture. UNCTAD.

Sojo, A. (2017). Protección social en América Latina: la desigualdad en el banquillo, Libros de la Cepal, № 143 (LC/PUB.2017/7-P). Comisión Económica para América Latina y el Caribe (Cepal).

Schlogl, L.; Sumner, A. (2014). How middle class are the «emerging middle» or «scooter class» in Indonesia? A household asset approach to social stratification, Economics and Development Studies Working Papers No. 201407, Bandung, Padjadjaran University.

UNICEF. (2012). Integrated social protection systems: Enhancing equity for children.

Vuolo, R. (2009). Social Exclusion Policies and Labour Markets in Latin America. En Hujo K., McClanahan S. (eds.) Financing Social Policy. Social Policy in a Development Context. Palgrave MacMillan. 